Arquivos Brasileiros de Educação Física

Brazilian Archives of Physical Education

ABEF

\title{
Mapeamento dos recursos financeiros utilizados para o Esporte de Alto Rendimento no Brasil
}

Mapping of the financial resources used for the Elite Sports in Brazil

Mapeo de los recursos financieros utilizados para el Deporte de Alto Rendimiento en Brasil

\section{Resumo}

Introdução: o fomento financeiro ao Esporte de Alto Rendimento (EAR) realizado historicamente por meio de isenções de taxas, tributos e impostos às próprias entidades esportivas, nos últimos anos foi somado a uma série de ações governamentais e pelo patrocínio de empresas privadas. Objetivo: identificar as fontes de recursos financeiros federais e privadas disponíveis para o EAR brasileiro, além de descrever os destinos e as formas de aplicação para tais recursos. Métodos: foi realizada uma análise documental, na Secretaria Nacional de Esporte de Alto Rendimento do Ministério do Esporte, em páginas eletrônicas oficiais e por meio de questionário eletrônicos para catalogar e categorizar dados sobre as fontes, os destinos e as formas de aplicação dos recursos financeiros investidos no esporte olímpico e paralímpico brasileiro. Resultados: evidenciou-se que o EAR brasileiro possui oito fontes de financiamento. Os recursos destas fontes podem ser destinados tanto para pessoas físicas como para pessoas jurídicas e aplicados de 13 formas diferentes: atletas, recursos humanos, treinamento, competição, material esportivo, equipamento esportivo, instalação esportiva, pesquisa científica, capacitação, tecnologia, despesa administrativa, avaliação da saúde e do desempenho, além dos investimentos com marketing. Conclusão: a categorização de informações do financiamento do EAR, apresentadas nesse trabalho, pode colaborar substancialmente com a avaliação e formulação de políticas e açooes governamentais, visto que a estratégia de identificação dos destinos e das formas de aplicação dos recursos financeiros pode colaborar com futuras pesquisas que objetivem avaliar os investimentos de duas ou mais fontes de recurso, simultaneamente, podendo-se determinar "o que" é investido e "como" os recursos são utilizados.

Palavras - chave: Esporte. Esporte de Alto Rendimento. Financiamento. Política Pública.

\begin{abstract}
Introduction: The financial support to the Elite Sports (ES), carried out historically through exemptions from fees and taxes to the sports entities, has been added in recent years to a series of governmental actions and sponsorship of private companies. Objective: To identify the sources of federal and private financial resources available to the Brazilian ES, in addition to describing the destinations and the forms of application for such resources. Methods: a documentary analysis was carried out in the National Secretariat of Elite Sports in the Ministry of Sports, in official electronic pages and by electronic questionnaire to catalog and categorize data on sources, destinations and ways of applying financial resources invested in the Olympic and Paralympic Brazilian sports. Results: it was evidenced that the Brazilian ES has eight sources of financing. The resources of these sources can be used both for individuals and legal entities and applied in 13 different ways: athletes, human resources, training, competition, sports equipment, sports materials, sports facilities, scientific research, professional qualification, technology, health and performance assessment, as well as marketing investments. Conclusion: the categorization of
\end{abstract}

1. Escola de Educação Física, Fisioterapia e Dança - Universidade Federal do Rio Grande do Sul - UFRGS, Porto Alegre, Rio Grande do Sul. Endereço para correspondência: Alan Ferreira, Rua Felizardo, 750, Bairro: Jardim Botânico, Escola de Educação Física, Fisioterapia e Dança - ESEFID/UFRGS, Porto Alegre, Rio Grande do Sul, CEP 90690 - 200, 3105.ferreira@gmail.com. Este conteúdo utiliza a Licença Creative Commons Attribution 4.0 International License Open Access. This content is licensed under a Creative Commons attribution-type BY. ISSN 2595 - 0096. 
information on ES funding presented in this paper can substantially contribute to the evaluation and formulation of government policies and actions, since the strategy of identifying destinations and ways of applying financial resources may contribute to future research that aim to evaluate the investments of two or more sources of resource, simultaneously, being able to determine "what is invested" and "how" the resources are used.

Keywords: Sport. Elite Sports. Financing. Public Policy.

\section{Resumen}

Introducción: el fomento financiero al Deporte de Alto Rendimiento (DAR) realizado históricamente por medio de exenciones de tasas, tributos e impuestos a las propias entidades deportivas, en los últimos años se ha sumado a una serie de acciones gubernamentales y por el patrocinio de empresas privadas. Objetivo: identificar las fuentes de recursos financieros federales y privados disponibles para el DAR brasileño, además de describir los destinos y las formas de aplicación para tales recursos. Métodos: se realizó un análisis documental, en la Secretaría Nacional de Deporte de Alto Rendimiento del Ministerio del Deporte, en páginas electrónicas oficiales y por medio de cuestionarios electrónicos para catalogar y categorizar datos sobre las fuentes, los destinos y las formas de aplicación de los recursos financieros en el deporte olímpico y paralímpico brasileño. Resultados: se evidenció que el DAR brasileño posee ocho fuentes de financiamiento. Los recursos de estas fuentes pueden ser destinados tanto para personas físicas y para personas jurídicas y aplicadas de 13 formas diferentes: atletas, recursos humanos, entrenamiento, competición, material deportivo, equipo deportivo, instalación deportiva, investigación científica, capacitación, tecnología, gasto administrativo, la evaluación de la salud y el rendimiento, además de las inversiones con marketing. Conclusión: la categorización de información del financiamiento del DAR, presentada en este trabajo, puede colaborar sustancialmente con la evaluación y formulación de políticas y acciones gubernamentales, ya que la estrategia de identificación de los destinos y de las formas de aplicación de los recursos financieros puede colaborar con futuras investigaciones que objetiven evaluar las inversiones de dos o más fuentes de recurso, simultáneamente, pudiéndose determinar "lo que" es invertido y "cómo" se utilizan los recursos.

Palabras - Clave: Deporte. Deporte de Alto Rendimiento. Financiación. Política Pública.

\section{INTRODUÇÃO}

\section{O financiamento do Esporte}

de Alto Rendimento (EAR) vem sendo discutido em vários estudos nacionais e internacionais como uma das estratégias políticas de promoção do esporte. Além disso, muitos trabalhos sugerem que a promoção de atletas de alto rendimento e os seus respectivos sucessos esportivos são resultados de um processo de planejamento estratégico ${ }^{1,2}$.

Tais estudos também têm demonstrado que os países necessitam, inevitavelmente, tratar seus investimentos de uma forma estratégica, ou seja, os investimentos, ao serem parte vital do sucesso esportivo, precisam estar em constante sintonia com os resultados obtidos (produto) ${ }^{2}$.

$$
\text { Neste contexto, o EAR }
$$
brasileiro, especialmente os esportes olímpicos e Paralímpicos, vem sendo considerado um dos setores estratégicos do Governo Federal, principalmente após a vitória do Rio de Janeiro para sediar os Jogos Olímpicos e Paralímpicos de 2016. Desta forma, para execução das políticas, o governo aloca recursos financeiros do Ministério do Esporte por meio da formalização de 
parcerias com outros entes públicos (municípios, estados, outros órgãos federais como universidades e ministérios) e entidades privadas sem fins lucrativos ${ }^{4}$.

Segundo De Bosscher et al. ${ }^{2}$, a criação de um ambiente favorável à evolução do desempenho e resultado esportivo está relacionada a um maior suporte de recursos financeiros que garanta a sustentabilidade de atletas e do sistema esportivo de um país. Porém, apesar de um razoável volume de trabalho sobre o financiamento do esporte brasileiro, não há na literatura científica um método estabelecido para o mapeamento do universo de fontes de recursos financeiros para o EAR brasileiro. A partir de tal mapeamento, informações de todas as fontes de recursos podem ser gerenciadas, permitindo a sua alocação estratégica.

Diante desta realidade e considerando que o apoio financeiro para o Esporte de Alto Rendimento pode criar mais oportunidades para os atletas desenvolverem o seu talento, este estudo tem o objetivo de identificar as fontes de recursos financeiros federais e privadas disponíveis para o EAR brasileiro, além de descrever os destinos e as formas de aplicação para tais recursos.

\section{MÉTODOS}

Para mapeamento das fontes de recursos financeiros do EAR brasileiro, este trabalho contemplou informações dos esportes olímpicos e paralímpicos dos Jogos Rio 2016, sendo realizado em três etapas: (1) definição do escopo de informações; (2) coleta de dados; (3) catalogação e categorizações dos dados sobre as fontes, os destinos e as formas de aplicação dos recursos. Foram realizadas: pesquisa documental, aplicação de questionários e preenchimento de formulários eletrônicos. Este trabalho foi aprovado pelo Comitê de Ética em Pesquisa com Humanos da Universidade Federal do Rio Grande do Sul, sob o parecer 2008010 .

Para definição do escopo de informações foi realizada pesquisa exploratória qualitativa, por meio da pesquisa documental, para identificar, conceituar e delimitar as fontes dos recursos financeiros disponíveis para o EAR no Brasil. Foram utilizados os métodos descritos por Ferreira $^{5}$ para catalogar e sistematizar as informações dessas fontes. Os dados catalogados sobre os recursos financeiros dizem respeito a sua natureza, sua origem, a identificação da fonte, seus possíveis destinos e suas formas de aplicação (Tabela 1). 
Tabela 1. Dados coletados para mapear as fontes de recursos financeiros do Esporte de Alto Rendimento no Brasil.

\begin{tabular}{c|l}
$\begin{array}{c}\text { Informação } \\
\text { coletada }\end{array}$ & \multicolumn{1}{c}{ Descrição } \\
\hline Natureza & $\begin{array}{l}\text { Natureza jurídica dos valores } \\
\text { investidos no Esporte de Alto } \\
\text { Rendimento }\end{array}$ \\
\hline Origem & $\begin{array}{l}\text { Origem dos recursos financeiros, } \\
\text { segundo sua natureza e sua } \\
\text { determinação orçamentária }\end{array}$ \\
\hline Fonte & $\begin{array}{l}\text { Instrumento legal que determina } \\
\text { a fonte de recursos públicos ou a } \\
\text { receita utilizada por entidades } \\
\text { privadas para financiamento do } \\
\text { Esporte de Alto Rendimento }\end{array}$ \\
\hline Destino & $\begin{array}{l}\text { Pessoas jurídicas e pessoas físicas } \\
\text { aptas a receber tais recursos }\end{array}$ \\
\hline Forma de & $\begin{array}{l}\text { Forma de uso final de utilização } \\
\text { dos recursos financeiros, dada } \\
\text { pelas pessoas jurídicas ou físicas, } \\
\text { destinatárias dos recursos } \\
\text { financeiros }\end{array}$ \\
\hline
\end{tabular}

Fonte: elaboração dos autores.

Considerou-se como escopo de informações os dados das entidades públicas e privadas, nacionais, estaduais e municipais que: administram a prática e as competições esportivas; que são fontes ou destinos de recursos financeiros aplicados no EAR; organizam ou promovem eventos esportivos; produzem ciência e tecnologia ligadas ao esporte; e, administram instalações esportivas.

Para obtenção das informações da Tabela 1, foi realizada pesquisa documental, preenchimento de formulários eletrônicos junto às confederações (Entidades Nacionais de Administração do Desporto) com objetivo de registrar os dados, classificálos, interpretá-los e analisá-los. Antes da padronização dos formulários e da aplicação dos questionários, foi realizado estudo piloto com entidades do atletismo e da natação paralímpica, para testagem e calibração dos instrumentos e procedimentos. Posteriormente, a coleta de dados foi dividida em quatro etapas:

(1) Obtenção de dados in loco no Ministério do Esporte: pesquisa documental em materiais impressos e eletrônicos, nos arquivos da Secretaria Nacional de Esporte de Alto Rendimento (SNEAR): foram consultados dados dos convênios, contratos de repasse e descentralizações firmados entre o ME - via SNEAR - e entidades públicas e privadas; projetos aprovados e incentivados pela Lei de Incentivo ao Esporte (LIE); lista de atletas contemplados pelo Programa Bolsa-Aleta e dos participantes do Plano Brasil Medalhas; além da lista de empresas estatais que investiram recursos financeiros no EAR.

(2) Obtenção de dados em páginas eletrônicas oficiais: pesquisa documental nas páginas eletrônicas oficiais das entidades que fazem parte do Sistema Nacional do Desporto ${ }^{6}$ para obter informações das entidades que organizam competições nacionais e internacionais e das entidades que patrocinam o EAR brasileiro; e, lista de escolas de educação física ligadas à 
instituições de ensino superior, públicas e privadas, no Brasil, inclusive sobre recursos oriundos da Lei Agnelo Piva, repassados para $\mathrm{COB}$ e para o $\mathrm{CPB}$, e destes para as confederações; e, sobre recursos financeiros privados oriundos da receita das confederações e de patrocínios de empresas privadas para essas entidades.

(3) Aplicação de questionário junto às confederações: questionário aplicado às 34 Entidades Nacionais de Administração do Desporto (ENADs) que administram esportes olímpicos e/ou paralímpicos. O questionário foi construído a partir das informações coletadas nas etapas anteriores, confeccionado e estruturado em três partes: a) apresentação; b) cinco perguntas sobre as caraterísticas do financiamento da entidade e de seus atletas; e c) estruturação das respostas (indicação dos atributos e dados necessários para a completa obtenção dos dados). As informações de todas as entidades e etapas foram catalogadas e compuseram o banco de dados final desse estudo.

(4) Catalogação e categorização dos dados: as informações das fontes, destinos e formas de aplicação dos recursos financeiros no EAR foram catalogadas e categorizadas segundo as premissas do modelo de gestão da informação proposto por Valentim?, adaptado ao contexto esportivo. Foram elaborados Glossário de Termos e Dicionário de Dados para orientar a catalogação e determinar a validação das informações quanto ao seu formato e conteúdo.

\section{RESULTADOS}

A pesquisa documental no Ministério do Esporte, nas páginas eletrônicas oficiais e a aplicação dos questionários permitiu detectar que o universo de recursos financeiros que financia o Esporte de Alto Rendimento brasileiro, considerando os recursos públicos federais e os privados, abrange:

(1) Recursos públicos federais oriundos do Orçamento do Ministério do Esporte como convênios e contratos de repasse e das Descentralizações (Decreto $\mathrm{n}^{\mathrm{o}}$ 6.170, de 2007), do Programa Bolsa-Atleta (Lei 10.891/2004) e do Programa Brasil Medalhas (Lei 12.395/2011);

(2) Recursos oriundos do patrocínio de Empresas Estatais;

(3) Das Loterias por meio da Lei Agnelo Piva (Lei 10.264/2001) Lei 11.438/2006;

(4) Da Lei de Incentivo ao Esporte;

(5) Dos recursos próprios oriundos da receita de Entidades 
Nacionais de Administração do

Desporto (Confederações); e,

(6) De patrocínios de empresas privadas para entidades esportivas.

A partir da identificação da fonte de recursos financeiros do EAR e do estudo dos dados coletados, foi possível identificar seus destinos (para entidades e pessoas físicas) e suas formas de aplicação (Figura 1, ver final do documento). Já o conceito e a descrição de cada fonte, de cada destino e de cada formas de aplicação dos recursos pode ser observado na Tabela 2 (vide final do documento). Detectou-se que os recursos das fontes supracitadas podem ser recebidos por seis destinos diferentes e, por estes, aplicados de 13 formas diferentes (Tabela 2). Destaca-se que a categorização aqui apresentada se baseia na utilização real dos recursos financeiros, realizada nos dois últimos ciclos olímpicos (2009 a 2012 e 2013 a 2016), conforme dados coletados no Ministério do Esporte. Importa salientar que conhecer "o que" (fontes) é investido e "como" (destino e forma de aplicação) os recursos financeiros são realmente utilizados pode colaborar com a qualificação da tomada de decisão de gestores, melhorar a eficiência das políticas esportivas, ou seja, melhorar a relação entre os investimentos e os resultados desejados.
Ressalta-se ainda que o método de categorização das fontes, dos destinos e das formas de aplicação dos recursos desenvolvida neste trabalho permite o estudo comparativo e simultâneo de todas as fontes de recursos públicos federais e privados disponíveis para o EAR brasileiro, possibilitando o monitoramento do uso de tais recursos no futuro, assim como da avaliação da eficácia das formas de destinação e aplicação realizadas do passado.

\section{DISCUSSÃO}

$\mathrm{Na}$ literatura internacional os recursos financeiros são tratados como o suporte inicial, de entrada de um sistema esportivo. Pesquisas anteriores indicam claramente que os países que mais investem no esporte de elite criam mais oportunidades para os atletas alcançarem o sucesso. Por exemplo, Green e Oakley ${ }^{8}$ encontraram uma relação linear entre o dinheiro gasto e as medalhas totais conquistadas pela Austrália desde a década de 1980. Segundo De Bosscher et al. ${ }^{2}$, o melhor preditor de sucesso nos resultados esportivos parece ser a quantidade absoluta de recursos financeiros alocados no esporte de elite.

Neste contexto, pesquisas têm apontado para a elevada importância da participação do Estado no apoio e financiamento das atividades esportivas que garantam a formação e o 
desenvolvimento de atletas. $\mathrm{O}$ desenvolvimento esportivo de um país parece estar fortemente relacionado com este fator, como demonstram os casos das assim chamadas potências esportivas ou em desenvolvimento, tais como: Austrália, China, Cuba, França, Rússia, Alemanha e Espanha, ${ }^{2,9}$.

No Brasil, a partir de 2004, o fomento financeiro ao Esporte de Alto Rendimento realizado historicamente por meio de isenções de taxas, tributos e impostos às próprias entidades esportivas, foi somado a uma série de ações governamentais, principalmente na esfera federal, com destaque aos convênios (Decreto 6.170, de 25 de julho de 2007 e Portaria Interministerial 507 , de 24 de novembro), a concessão de bolsas para atletas (Lei 10.892, de 9 de julho de 2004) e, a Lei de Incentivo ao Esporte (11.438, de 29 de dezembro de 2006) ${ }^{4}$.

Contudo, mesmo diante desta gama de fontes de financiamento e sabendo-se que os financiadores das atividades esportivas são principalmente as pessoas jurídicas de direito público, dentre elas as empresas estatais e os órgãos do setor público, que por meio de políticas e ações destinam recursos financeiros ao $\mathrm{EAR}^{9}$, até a presente pesquisa não haviam informações sistematizadas sobre os destinos e as formas de aplicação dos recursos disponíveis para o esporte de elite brasileiro.

Este trabalho complementa o estudo de Ferreira et al. ${ }^{4}$, quando os pesquisadores mapearam somente os destinos e as formas de aplicação dos recursos oriundos dos convênios (Decreto 6.170, de 25 de julho de 2007) investidos em esportes paralímpicos, visto que as fontes, os destinos e as formas de aplicação apresentadas no presente estudo abrange todos os esportes e todas as fontes de financiamento do EAR brasileiro.

Trabalhos em várias partes do mundo vêm sendo realizadas na tentativa de compreender o funcionamento dos sistemas esportivos e os fatores que interferem no sucesso do esporte de elite de diferentes países ${ }^{2,8,10,11,12}$. Àqueles que analisam as estruturas esportivas dos países coletam informações para, de maneira sistematizada, compreender as variáveis ou dimensões que envolvem os seus sistemas esportivos. Por isso, conhecer detalhadamente o financiamento do EAR no Brasil pode colaborar profundamente com a gestão dos recursos, consequentemente, com o monitoramento de sua destinação e de sua aplicação.

Segundo Mazzei et al. ${ }^{13}$, há subaproveitamento dos recursos 
financeiros disponíveis para o esporte brasileiro, assim como inexistência de metas e controle de dos investimentos. Diante desta realidade, este estudo colabora com o conhecimento e com a gestão do Esporte de Alto Rendimento no país, tornando mais claras as explicações sobre os investimentos realizados em atletas e entidades.

\section{CONCLUSÃO}

O presente trabalho evidenciou que o Esporte de Alto Rendimento (EAR) brasileiro possui oito fontes para seu financiamento. Os recursos destas fontes podem ser destinados tanto para pessoas físicas como para pessoas jurídicas e, por estes aplicados de 13 formas diferentes.

$$
\text { Após categorização das }
$$

informações das fontes de financiamento do EAR, evidenciou-se que os recursos podem ser aplicados em Atletas, Recursos humanos, Treinamento, Competição, Material esportivo, Equipamento esportivo, Instalação esportiva, Pesquisa científica, Capacitação, Tecnologia, Despesa administrativa, Avaliação da saúde e do desempenho, além dos investimentos com Marketing.

O Brasil tem investido recursos financeiros no esporte de alto rendimento com maior intensidade nos últimos anos, com o objetivo de ter sucesso e melhores resultados esportivos em grandes eventos internacionais. Uma das estratégias adotadas pelo governo brasileiro é o repasse de recursos financeiros para organizações como as entidades que administram os esportes olímpicos e Paralímpicos, assim como para outros entes públicos. No entanto, muitas vezes os recursos financeiros são dispendidos sem classificação das ações, entidades ou público a que são destinados. Sendo assim, a sistematização dessas informações, apresentadas nesse trabalho, pode colaborar substancialmente com a avaliação e formulação de políticas e ações governamentais.

Adicionalmente, a estratégia utilizada nesse estudo - identificação dos destinos e das formas de aplicação dos recursos financeiros - pode colaborar com futuras pesquisas que objetivem avaliar os investimentos de duas ou mais fontes de recurso, simultaneamente, podendo-se determinar "o que" é investido e "como" os recursos são utilizados.

\section{REFERÊNCIAS}

1. Andersen S, Ronglan LT (Eds.). Norway: AIT Otta AS. Sport for all and elite sport: Do they benefit one another? In $\mathrm{NOC}^{*} \mathrm{NSF}$ (Ed.), proceedings of the 9th World Sport for All Congress, 'Sport for all and elite sport: Rivals or partners?' p. 25, NOCNSF, Ahrnem, NL, 2012.

2. De Bosscher V, Shibli S, Westerbeek 
H, Van Bottenbrug M. Successful Elite Sport Policies: an international comparison of the Sports Policy factors Leading to Internacional Sporting Success (SPLISS 2.0) in 15 nations. Meyer \& Meyer Sports (UK) Ltd. Reino Unido, 2015.

3. Brasil. Lei no 12.798 , de 4 de abril de 2013b. Estima a receita e fixa a despesa da União para o exercício financeiro de 2013. Disponivel em: http://www.planalto.gov.br/ccivil_03/_At o2011-2014/2013/Lei/L12798.htm.

Acesso em: 14 jul. 2018.

4. Ferreira ACD, Vitor KP, Haiachi MC, Reppold Filho AR. Financing of the Paralimpic Sports In Brazil: Agreements. Brazilian Journal of Education, Technology and Society (BRAJETS) - Especial Section, "Disability, Education, Technology and Sport”, Vol. 11, No. 01, Jan-Mar, 2018.

5. Ferreira ACD. Um método para a gestão de informações do Esporte de Alto Rendimento no Brasil. 2018. $503 \mathrm{f}$. Tese (Doutorado em Ciências do Movimento Humano) - Escola de Educação Física, Fisioterapia e Dança, Universidade Federal do Rio Grande do Sul, Porto Alegre, 2018.

6. Brasil. Lei n ${ }^{\circ} 9.615$ de 24 de março de 1998. Institui normas gerais sobre desporto e dá outras providências. Diário Oficial da República Federativa do Brasil. Brasília DF, 25 de março de 1998.

7. Valentim MLP. Gestão da informação e do conhecimento no âmbito da Ciência da Informação. São Paulo: Polis: Cultura Acadêmica, 2008.

8. Green M, Oakley B. Elite sport development systems and playing to win: uniformity and diversity in international approaches. Leisure Studies. v.20, p. 247-267, 2001.
9. Ferreira R. Políticas para o Esporte de Alto Rendimento - Estudo Comparativo de alguns Sistemas Esportivos Nacionais visando um Contributo para o Brasil. São Paulo, 2007.

10. Digel HBA. Comparison of competitive sport systems. New Studies in Athletics. v.17, 1, p. 37-50, 2002.

11. De Bosscher V, Bingham J, Shibli S, Van Bottenburg M, De Knop P. The global sporting arms race. An internacional comparative study on sports Policy factors leading to international sporting success. Oxford: Meyer \& Meyer Sport (UK), 2008.

12. Houlihan B, Green M. Comparative elite sport development: systems, structures and public policy. Elsevier. Burlington, 2008.

13. Mazzei LC, De Barros MT, Da Cunha BF, Silveira BMT, De Bosscher, V. High performance sport in Brazil. Structure and policies comparison with the international contexto. Gestión y Política Pública, pp. 83-111, 2015.

Informações do artigo / Information of this article:

Recebido: 01/12/2018

Aprovado: 23/12/2018

Publicado: 15/01/2019

Received: 01/12/2018

Approved: 23/12/2018

Published: 15/01/2019

Mrs. Alan de Carvalho Dias Ferreira ORCID: 0000-(0002-0139-4318

Contribuição dos autores

Alan de Carvalho Dias Ferreira participou do mapeamento dos dados e da produção do texto do artigo. 
Alberto Reinaldo Reppold Filho orientou o trabalho e revisou o texto final.

Conflito de interesses

Os autores declaram não haver conflito de interesses.

\section{Agradecimentos}

Os autores gostariam de agradecer à Fundação de Apoio da Universidade do Rio Grande do Sul e ao Ministério do Esporte por seu apoio durante o desenvolvimento do trabalho.

\section{Financiamento}

Este trabalho foi apoiado e financiado pelo Ministério do Esporte, conforme convênio número 58.701.004100.201230 .

Como citar este artigo:

Ferreira, A. C. D.; Reppold Filho, A. R. Mapeamento dos recursos financeiros utilizados para o Esporte de Alto Rendimento no Brasil. Arq. Bras. Ed. Fís., Tocantinópolis, v. 1, n.2, Ago./Dez., p. 69 - 81, 2018. 
Figura 1. Modelo esquemática das fontes, dos destinos e das formas de aplicação dos recursos financeiros disponíveis para o Esporte de Alto Rendimento brasileiro.

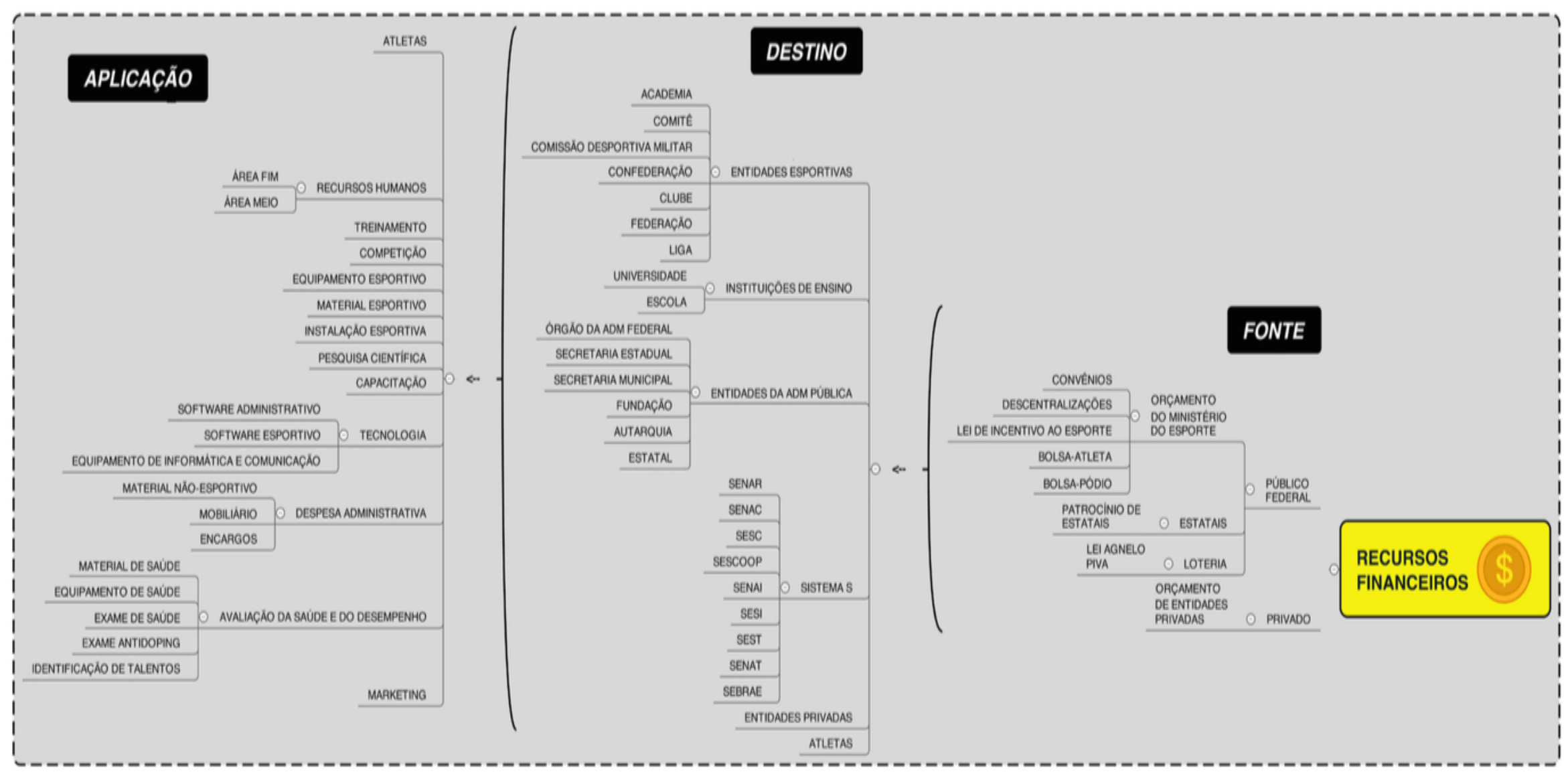

Fonte: elaboração dos autores. 
Tabela 2. Dados coletados para mapear as fontes de recursos financeiros do Esporte de Alto Rendimento no Brasil.

\begin{tabular}{|c|c|}
\hline \multicolumn{2}{|r|}{ FONTES } \\
\hline Convênios & $\begin{array}{l}\text { Instrumento que disciplina a transferência de recursos financeiros de dotações Orçamentárias da União e tem como partícipe, de um lado, órgão ou entidade da } \\
\text { administração pública federal e, de outro lado, órgão ou entidade da administração pública estadual, distrital ou municipal, direta ou indireta, ou ainda, } \\
\text { entidades privadas sem fins lucrativos, em regime de mútua cooperação. Também pode ser realizado com a intermediação (contrato de repasse) de uma } \\
\text { instituição ou agente financeiro público federal, que representa a União na execução e fiscalização da transferência (Decreto no } 6.170 \text {, de 2007). }\end{array}$ \\
\hline Descentralizações & $\begin{array}{l}\text { Descentralização de crédito entre órgãos e/ou entidades integrantes do Orçamento da União, para execução de ações de interesse da unidade orçamentária } \\
\text { descentralizadora. Movimentação de parte do orçamento, mantidas as classificações institucional, funcional, programática e econômica, para que outras } \\
\text { unidades administrativas possam executar a despesa orçamentária. (Decreto no } 6.170 \text {, de } 2007 \text { ) }\end{array}$ \\
\hline Bolsa-Atleta & Repasse de recursos públicos federais diretamente para atletas habilitados por resultados esportivos (Lei 10.891/2004). \\
\hline $\begin{array}{l}\text { Plano Brasil } \\
\text { Medalhas }\end{array}$ & $\begin{array}{l}\text { Repasse de recursos públicos federais diretamente para atletas habilitados ao Bolsa Pódio, além de recursos para viabilização de equipe técnica multidisciplinar; } \\
\text { para participação em competições internacionais; para realização de treinamentos e intercâmbios internacionais; e, para fornecimento de equipamentos e } \\
\text { materiais esportivos de alta performance (Lei 12.935/2011). }\end{array}$ \\
\hline $\begin{array}{l}\text { Lei de Incentivo ao } \\
\text { Esporte }\end{array}$ & $\begin{array}{l}\text { Investimento de parte do Imposto de Renda de empresas e pessoas físicas em projetos esportivos aprovados pelo Ministério do Esporte. Empresas podem } \\
\text { investir até } 1 \% \text { do imposto e as pessoas físicas até } 6 \% \text {. (Lei } 11.438 / 2006 \text { ). }\end{array}$ \\
\hline $\begin{array}{l}\text { Patrocínio de } \\
\text { Estatais }\end{array}$ & $\begin{array}{l}\text { Investimentos de empresas estatais (Petrobras, Infraero, Eletrobras, Correios, Caixa Econômica Federal, Banco Nacional de Desenvolvimento Econômico e } \\
\text { Social (BNDES), Banco do Brasil e Banco do Nordeste) para confederações, federações e clubes. }\end{array}$ \\
\hline $\begin{array}{c}\text { Loterias - Lei } \\
\text { Agnelo Piva }\end{array}$ & $\begin{array}{l}\text { Investimento de } 2 \% \text { da arrecadação bruta de todas as loterias federais, repassados ao Comitê Olímpico Brasileiro e ao Comitê Paraolímpico Brasileiro (Lei } \\
10.264 / 2001) \text {. }\end{array}$ \\
\hline Entidades privadas & Oriundos da receita de Entidades de Administração do Esporte e de patrocínios de empresas privadas. \\
\hline \multicolumn{2}{|r|}{ DESTINOS } \\
\hline Atletas & Quando os recursos são repassados das fontes diretamente para atletas. \\
\hline Entidades Esportivas & $\begin{array}{l}\text { Quando os recursos saõ repassados para entidades com atividade principal ligada ao esporte, como comitês, confederações, federações, clubes, ligas, academias } \\
\text { e comissões esportivas. }\end{array}$ \\
\hline $\begin{array}{l}\text { Instituições de } \\
\text { Ensino }\end{array}$ & $\begin{array}{l}\text { Quando os recursos são repassados para universidades, faculdades, centros ou institutos de ensino superior, públicos ou privados; para escolas de ensino médio } \\
\text { ou fundamental, públicas e privadas. }\end{array}$ \\
\hline $\begin{array}{l}\text { Entidades da } \\
\text { Administração } \\
\text { Pública }\end{array}$ & $\begin{array}{l}\text { Quando os recursos são repassados para entidades que integram a administração pública direta ou indireta, como ministérios, secretarias e outros órgãos do } \\
\text { poder executivo, autarquias e fundações públicas. }\end{array}$ \\
\hline Empresas Privadas & Quando os recursos são repassados para pessoas jurídicas de direito privado, com ou sem fins lucrativos. \\
\hline $\begin{array}{l}\text { Entidades do } \\
\text { Sistema S }\end{array}$ & $\begin{array}{l}\text { Quando os recursos são repassados a uma ou ao conjunto de organizações das entidades corporativas voltadas para o treinamento profissional, assistência social, } \\
\text { consultoria, pesquisa e assistência técnica, como o Serviço Nacional de Aprendizagem Industrial (Senai); Serviço Social do Comércio (Sesc); Serviço Social da } \\
\text { Indústria (Sesi), etc. }\end{array}$ \\
\hline \multicolumn{2}{|r|}{ FORMAS DE APLICAÇÃO } \\
\hline Atleta & Investimentos diretos para atletas, como bolsas, salários, ajuda de custos. \\
\hline Recursos Humanos & $\begin{array}{l}\text { Investimentos com bolsas, salários, ajuda de custos - na forma de auxílio financeiro - para gestores, árbitros, técnico esportivo, treinadores, equipe } \\
\text { multidisciplinar ligados à área fim ou à ára meio. }\end{array}$ \\
\hline Treinamento & $\begin{array}{l}\text { Investimentos para participação de atletas e membros da equipe multidisciplinar em treinamentos nacionais e internacionais, como passagens aéreas, } \\
\text { documentação, transporte, alimentação, hospedagem, inscrições e seguros. }\end{array}$ \\
\hline
\end{tabular}




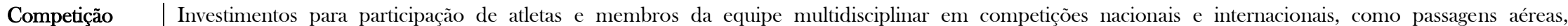
Continuação ... $\quad$ documentação, transporte, alimentação, hospedagem, inscrições e seguros.

Material Esportivo $\quad$ Investimentos para aquisição ou manutenção de objeto ou conjunto de objetos (materiais) empregados em um ou vários esportes, considerados de consumo.

Equipamento $\quad$ Investimentos para aquisição ou manutenção de aparelho ou conjunto de objetos (equipamentos) empregados em um ou vários esportes, considerados

Esportivo

Instalação Esportiva

Pesquisa Científica

Capacitação

Investimentos com cursos e capacitação de atletas, treinadores, gestores, árbitros e equipe multidisciplinar.

Investimentos com o desenvolvimento ou aquisicão de programas destinados a avaliação de atletas e/ou gestão de treinamentos/competiçoes; com a aquisicão de hardware (computador, tablet, impressoras, scanners, projetores, redes, celulares, rádio-comunicadores); com a aquisição de programas destinados a gestão administrativa de entidades esportivas.

Despesa

Despesas com serviços, locação e aquisição de material de expediente, além de despesas com premiações como medalhas, troféus, etc; aquisição de mobiliário

Despes

Administrativa

Avaliação da Saúde

e do Desempenho

depreciacão (do valor de um bem permanente), contribuicões (sindicais e de classe, etc.).

Investimentos destinados para avaliação, prescrição e tratamentos de atletas; investimentos com materiais não-esportivos, utilizados por profissionais de saúde

Investimentos destinados para avaliação, prescrição e tratamentos de atletas; investimentos com materiais não-esportivos, utilizados por profissionais de saúde
como médicos, fisioterapeutas, fisiologistas, nutricionistas, etc; investimentos para a aquisição de equipamentos não-esportivos, utilizados por profissionais de saúde como médicos, fisioterapeutas, inclusive para avaliação e testes de atletas; despesas com exames ou avaliações de atletas, exames antidoping, realizadas por entidades credenciadas para esse fim; investimentos com testes e avaliações para identificação e desenvolvimento de talentos esportivos

Marketing $\quad$ Investimentos com publicidade, propaganda, promoção de vendas, merchandising, relações públicas, marketing pessoal, venda pessoal e marketing esportivo,

Fonte: elaboração dos autores. o é, com o conjunto de processos que envolvem a criação, a comunicação e a entrega de valor para os clientes. 